\title{
Novel GFM2 variants associated with early-onset neurological presentations of mitochondrial disease and impaired expression of OXPHOS subunits
}

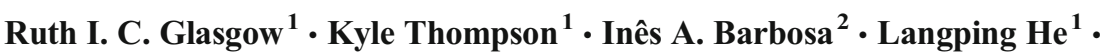 \\ Charlotte L. Alston ${ }^{1} \cdot$ Charu Deshpande ${ }^{2} \cdot$ Michael A. Simpson ${ }^{2}$. \\ Andrew A. M. Morris ${ }^{3,4}$ • Axel Neu ${ }^{5}$. Ulrike Löbel ${ }^{6}$ - Julie Hall ${ }^{7}$ Holger Prokisch ${ }^{8,9}$. \\ Tobias B. Haack $^{9,10}$ • Maja Hempel $^{11}$ • Robert McFarland ${ }^{1} \cdot$ Robert W. Taylor $^{1}$
}

Received: 22 September 2017 / Accepted: 3 October 2017 /Published online: 26 October 2017

(C) The Author(s) 2017. This article is an open access publication

\begin{abstract}
Mitochondrial diseases are characterised by clinical, molecular and functional heterogeneity, reflecting their bigenomic control. The nuclear gene GFM2 encodes mtEFG2, a protein with an essential role during the termination stage of mitochondrial translation. We present here two unrelated patients harbouring different and previously unreported compound heterozygous (c.569G $>$ A, p.(Arg190Gln); c.636delA, p.(Glu213Argfs*3)) and homozygous (c.275A>C, p.(Tyr92Ser)) recessive variants in GFM2 identified by whole exome sequencing (WES) together with histochemical and biochemical findings to support the diagnoses of pathological GFM2 variants in each case. Both patients presented similarly in early childhood with global developmental delay, raised CSF lactate and abnormalities on cranial MRI. Sanger sequencing of familial samples confirmed the segregation of bi-allelic GFM2 variants with disease, while investigations into steady-state mitochondrial protein levels revealed respiratory chain subunit defects and loss of mtEFG2 protein in muscle. These data
\end{abstract}

Robert W. Taylor

robert.taylor@ncl.ac.uk

1 Wellcome Centre for Mitochondrial Research, Institute of Neuroscience, The Medical School, Newcastle University, Newcastle upon Tyne NE2 4HH, UK

2 Department of Medical and Molecular Genetics, King's College London School of Medicine, London, UK

3 Division of Evolution and Genomic Sciences, School of Biological Sciences, University of Manchester, Manchester, UK

4 Alder Hey Children's Hospital NHS Foundation Trust, Liverpool, UK

5 University Children's Hospital, University Medical Center Hamburg-Eppendorf, Hamburg, Germany demonstrate the effects of defective mtEFG2 function, caused by previously unreported variants, confirming pathogenicity and expanding the clinical phenotypes associated with GFM2 variants.

Keywords WES - GFM2 - Mitochondrial translation · Developmental delay $\cdot$ Mitochondrial disease

\section{Introduction}

Mitochondrial disorders are a genetically and clinically heterogeneous group of diseases which can arise due to defects in the oxidative phosphorylation (OXPHOS) system. The OXPHOS system comprises five multi-subunit protein complexes - the electron transport chain (complexes I-IV) and ATP synthase (complex V)-which function together to generate cellular energy in the form of ATP. Mitochondria possess

6 Department of Diagnostic and Interventional Neuroradiology, University Medical Center Hamburg-Eppendorf, Hamburg, Germany

7 Department of Neuroradiology, Royal Victoria Infirmary, Newcastle upon Tyne, UK

8 Institute of Human Genetics, Helmholtz Zentrum München, Oberschleißheim, Germany

9 Institute of Human Genetics, Technische Universität München, Munich, Germany

10 Institute of Medical Genetics and Applied Genomics, University of Tübingen, Tübingen, Germany

11 Institute of Human Genetics, University Medical Center Hamburg-Eppendorf, Hamburg, Germany 
their own circular, double-stranded 16,569 bp genome, encoding 22 transfer RNAs (tRNAs), 2 ribosomal RNAs (rRNAs) and 13 polypeptides [1]. All OXPHOS components, with the exception of complex II, are under bi-genomic control and as such are the product of both nuclear DNA and mitochondrial DNA (mtDNA)-encoded proteins [2]. Nuclear genes also encode the proteins responsible for mtDNA maintenance, mitochondrial transcription and translation and all other mitochondrial processes. As a consequence, mutations in either mitochondrial or nuclear genes may compromise ATP synthesis and cause mitochondrial disease. Extensive clinical and genetic heterogeneity makes the identification, characterisation and diagnosis of mitochondrial disease challenging, because clinical features often overlap with other neurological or systemic diseases. The advent of nextgeneration sequencing, specifically whole exome sequencing (WES), has improved the identification of disease-causing pathogenic variants in many different genes resulting in a much greater diagnostic yield than previous candidate gene screening approaches [3].

Mutations in genes involved in the translation of mtDNA are well-documented causes of mitochondrial disease [4, 5]. These genetic variants characteristically lead to combined OXPHOS deficiencies. The spectrum of resulting clinical phenotypes is broad, often manifesting as multi-system disease with the heart, skeletal muscle, brain and liver commonly affected [6]. The translational machinery within mitochondria is distinct to that of the cytosol and has several features which are reminiscent of the system employed in prokaryotic protein synthesis. This is reflective of the evolutionary origins of mitochondria as an endosymbiont $\alpha$-proteobacterium [7]. Mitochondria possess their own mitoribosome which is a combination of $12 \mathrm{~S}$ and $16 \mathrm{~S}$ rRNAs, encoded within the mitochondrial genome, and at least 80 nuclear genes encode the proteins that form the large and small mitoribosomal subunits [8].

Pathogenic variants have been described in several of the mitoribosomal subunits [9-11]. Mitochondrial translation defects can also arise due to mutations in the mitochondrial tRNAs and in the mitochondrial protein translation factors [12]. Mitochondrial amino-acyl tRNA synthetases (mtaaRSs) are a group of enzymes with an essential role within mitochondrial protein synthesis and are associated with a continuously expanding number of mitochondrial disease presentations. There are $19 \mathrm{mt}-$ aaRSs, which utilise ATP to specifically catalyse the attachment of amino acids to their cognate tRNA [13]. Mitochondrial diseases resulting from mutations in genes encoding all 19 of the mt-aaRSs have now been described in the literature, with the most recently identified being WARS2 [14].

The human genes GFM1 and GFM2 encode the proteins mtEFG1 and mtEFG2, respectively, both of which are homologs of the highly conserved bacterial translation elongation factor $\mathrm{G}$ (EF-G) [15]. EF-G functions at multiple stages within prokaryotic protein synthesis, with roles in the translocation of the ribosome during elongation and ribosome recycling upon termination or stalling of translation [16]. In human mitochondria, the dual roles of prokaryotic EF-G are carried out by the two distinct human homologs. Human mtEFG1 acts as an elongation factor with mitoribosome translocation activity, while $\mathrm{mtEFG} 2$ functions at the termination step of translation to disassemble the mitoribosome and allow subsequent cycles of mitochondrial protein synthesis [17].

Seventeen mitochondrial disease patients with GFM1 mutations across 13 families have been described in the literature so far. Earlier cases were associated with very severe systemic disease with early or neonatal onset, resulting in death within the first 2 years of life. Some clinical features, such as microcephaly, liver disease and encephalopathy, were common to multiple cases. However, more recent publications have described a new, milder, disease progression associated with GFM1 mutations, with survival at 6 and 7 years of age $[18,19]$.

Two families with gene defects in GFM2, identified through whole exome sequencing, have previously been described in the literature associated with Leigh syndrome, microcephaly, simplified brain gyral pattern and insulindependent diabetes [20,21]. Here we present two unrelated patients with previously unreported variants in GFM2, documenting OXPHOS deficiencies in different tissues and expanding the clinical phenotypes associated with GFM2-related mitochondrial disease.

\section{Patients and methods}

All studies were completed according to local Ethical Approval of the Institutional Review Boards of Newcastle University (the National Research Ethics Service Committee North East-Newcastle \& North Tyneside 1) and of the Technische Universität München. In agreement with the Declaration of Helsinki, all individuals or their guardians provided written informed consent before undergoing evaluation and genetic testing.

\section{Patient 1}

Patient 1, an 11-year-old male, is the first child of healthy nonconsanguineous parents and has a healthy younger brother. Pregnancy was complicated by intrauterine growth restriction and he was born at term, in good condition, by normal vaginal delivery weighing $2.0 \mathrm{~kg}$. He had asymptomatic hypoglycaemia in the neonatal period and mild jaundice and was tube fed initially. He subsequently had a urinary tract infection and required orchidopexy for an undescended right testis.

Developmental delay was first noted at 2.5 years in relation to language and communication skills; he had acquired his first words at $12-18$ months, but did not put words together 
until aged 3 years. From 5 years onwards, he has become increasingly dysarthric. He has used a knife and fork from 3 years of age but has never been able to write. He started walking at 14 months and could run at 4 years but he subsequently developed a dystonic posture of his right foot and spasticity in both legs, leading to toe walking and loss of ambulation at 8 years; he currently mobilises by crawling. He has been continent since 2 years of age and continues to make slow academic progress. He has a normal head circumference, normal vision and hearing and no involuntary movements or seizures.

Cranial MRI showed symmetrical bilateral high signal on T2-weighted images in the caudate, putamen and cerebellar dentate nucleus. There were also abnormalities in the corpus callosum and the subcortical white matter of the cerebral and, particularly, the cerebellar hemispheres, with further abnormal areas in the deep white matter (Fig. 1a, b). CSF lactate was elevated on two occasions at 3.2 and $3.4 \mathrm{mmol} / \mathrm{L}$ (normal range, $0.7-2.1 \mathrm{mmol} / \mathrm{L}$ ). Sequencing of the mitochondrial genome and the NFU1 gene were both normal.

\section{Patient 2}

Patient 2, a 7-year-old female, is the second child of consanguineous parents originating from Syria. Born following an uneventful pregnancy at full term, development was unremarkable in the first 2 years of life. At the age of 2 years and 2 months, involuntary movements of the left hand were reported and within a few months, these had extended to involve all four limbs. Muscle strength and mass deteriorated and she lost the ability to walk at 4 years, to sit at 5 years and subsequently lost the ability to speak. At the age of 6 years, she presented with her first seizure and has subsequently developed a severe epilepsy disorder. Clinical assessment reveals severe global developmental delay, myopathic facies with an open mouth appearance and drooling, severe axial hypotonia with hypertonic limbs and dystonic involuntary movements. Communication was restricted to phonetic reading.

Lactate was repeatedly elevated in both serum (up to $4.1 \mathrm{mmol} / \mathrm{L}$ ) and CSF (up to $3.1 \mathrm{mmol} / \mathrm{L}$ ). Cranial MRI showed diffuse hyperintensities on T2-weighted imaging of
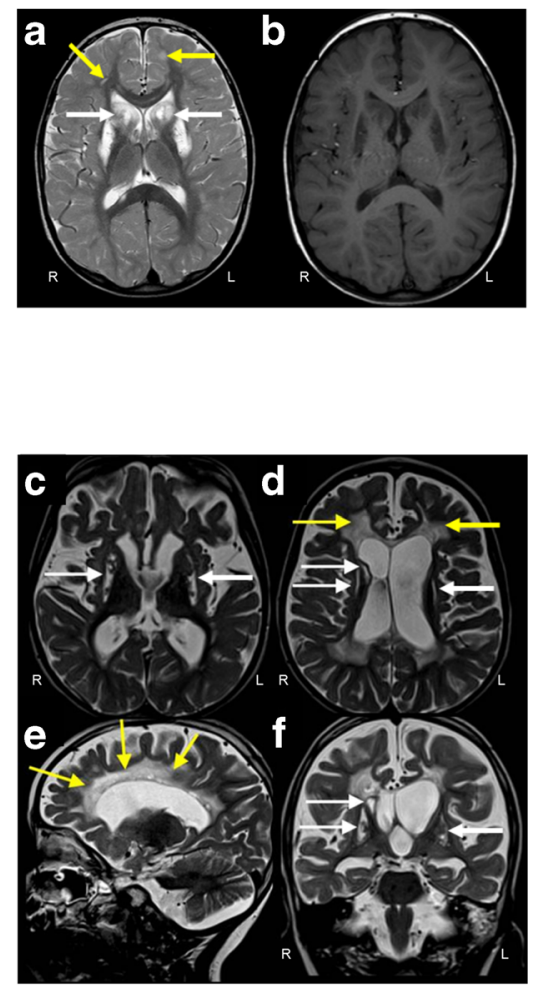

Fig. 1 Cranial MRI, histochemical and biochemical investigations. MRI of Patient 1 showing bilateral T2 hyperintensities involving supratentorial white matter (yellow arrows), head of caudate nucleus (white arrows), putamen and genu and splenium of the corpus callosum (a) characterised by low T1 signal suggesting irreversible tissue damage (b). MRI of Patient 2 showing extensive T2 hyperintensities associated with volume loss involving bilateral periventricular and central white matter $(\mathbf{d}, \mathbf{e}$, yellow arrows) and defects involving both putamina and the head of caudate nucleus on the right (c, $\mathbf{d}, \mathbf{f}$, white arrows). Right (R) and left
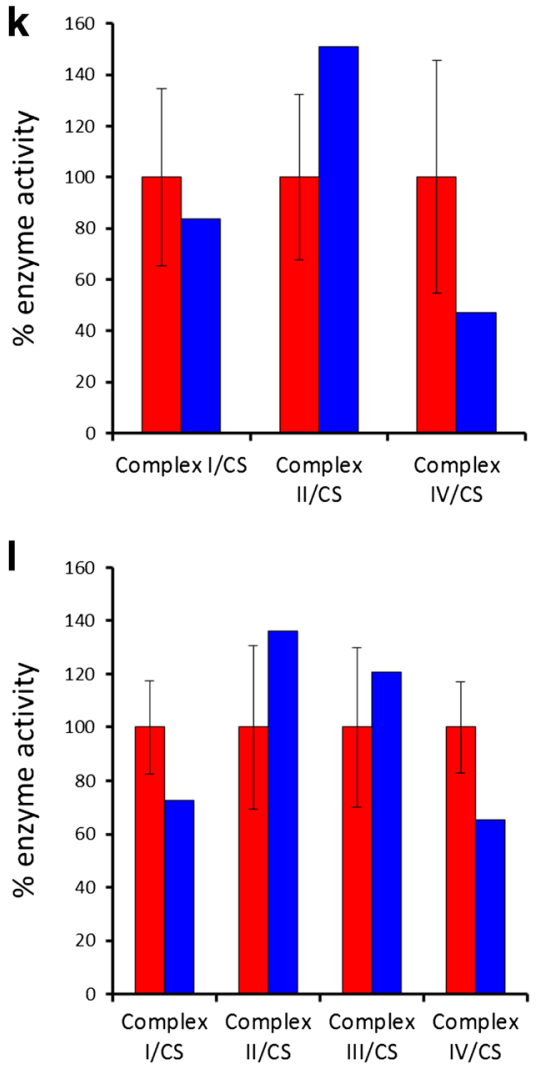

(L) are indicated. (g) Haematoxylin and eosin stain shows normal skeletal muscle morphology. Succinate dehydrogenase (SDH) (h), cytochrome $c$ oxidase $(\mathrm{COX})(\mathbf{i})$ and COX-SDH histochemistry (j) reveal a generalised and widespread COX deficiency. Respiratory chain enzyme activity measurements in skeletal muscle (k) and fibroblasts (I) demonstrate a severe complex IV defect in skeletal muscle and a mild complex I and IV defect in Patient 1 fibroblast cells compared to age-matched controls (red: controls, blue: patient 1 ) 
the periventricular and central white matter with associated volume loss and atrophy of corpus callosum as well as T2 hyperintense defects of bilateral putamen and head of caudate nucleus (Fig. 1c-f). EEG revealed multifocal seizure activity.

\section{Histopathology and biochemistry}

A diagnostic skeletal muscle biopsy was obtained from Patient 1 with informed consent; histological and histochemical investigations were carried out following standard procedures. The biochemical assessment of respiratory chain enzyme activities was undertaken as previously described in a skeletal muscle homogenate [22].

\section{Molecular genetic studies}

WES was carried out for both patients to identify candidate pathogenic variants. Methodologies and variant filtering pipelines were carried out as previously described in $[23,24]$ for Patient 1 and Patient 2, respectively. Variants with a minor allele frequency (MAF) $\geq 0.01(1 \%)$ on external sequencing databases such as the Genome Aggregation Database (gnomAD), and in-house control data sets, were excluded from the variant pool. Autosomal recessive variants (either compound heterozygous or homozygous) in genes known to encode proteins with mitochondrial function were then prioritised for further investigation.

Sanger sequencing of amplified gene products was carried out in accordance with BigDye ${ }^{\circledR}$ Terminator v3.1 Cycle Sequencing Kit manufacturing protocol. Sequencing primers for each variant were designed, using Primer3 (http://primer3. ut.ee), and sequences are available on request.

\section{Sequence analysis and in silico pathogenicity prediction}

Sequence data was viewed and analysed using FinchTV. Variants are named according to transcript variant 1 of GFM2 (GenBank accession number: NM_032380.4). Multiple sequence alignments were carried out using SeaView. Four freely available web-based programmes, Align GVGD (http://agvgd.iarc.fr/agvgd_input.php), SIFT (http://sift.jcvi.org), PolyPhen (http://genetics.bwh.harvard. edu/pph2/) and Combined Annotation Dependent Depletion (CADD) (http://cadd.gs.washington.edu/home), were used to predict the pathogenicity of the missense amino acid changes present in each patient through the analysis of Grantham differences and sequence homology.

\section{Cell culture}

Primary fibroblast cell lines for both patients and paediatric controls were incubated at $37{ }^{\circ} \mathrm{C}$ in a humidified $5 \% \mathrm{CO}_{2}$ atmosphere and grown in $75-\mathrm{cm}^{2}$ flasks of $12 \mathrm{~mL}$ Gibco®
MEM (minimum essential medium) containing $1 \mathrm{mM}$ pyruvate, $2 \mathrm{mM}$ L-glutamine and $4500 \mathrm{mg} / \mathrm{L}$ glucose, and supplemented with $10 \%$ FCS, $1 \times$ non-essential amino acids, $1 \times$ penicillin/streptomycin and $50 \mu \mathrm{g} / \mathrm{mL}$ uridine.

\section{Sample preparation, SDS-PAGE and Western blot analysis}

Fibroblast cell pellets were re-suspended in lysate buffer (50 mM Tris/HCl pH 7.4, $130 \mathrm{mM} \mathrm{NaCl}, 2 \mathrm{mM} \mathrm{MgCl}_{2}$, $1 \mathrm{mM}$ PMSF, $1 \%$ Nonidet P-40, $1 \times$ EDTA-free protease inhibitor), vortexed for $30 \mathrm{~s}$ and centrifuged at $1000 \mathrm{~g}$ for $2 \mathrm{~min}$, retaining the supernatant. Skeletal muscle $(10-15 \mathrm{mg})$ was frozen in liquid nitrogen, then powdered and suspended in $1 \mathrm{~mL}$ RIPA buffer (1\% IGEPAL, $0.5 \%$ sodium deoxycholate, $0.1 \%$ SDS, $1.5 \%$ Triton X-100, 10 mM beta mercaptoethanol, protease inhibitor (Pierce) and $1 \mathrm{mM}$ PMSF in PBS). The muscle homogenate was then vortexed, incubated on ice for $45 \mathrm{~min}$ and subjected to $2 \times 5$-s homogenisations in a polytron tissue homogeniser. The final muscle lysates were prepared by centrifugation at $14,000 \mathrm{~g}$ for $10 \mathrm{~min}$ at $4{ }^{\circ} \mathrm{C}$, retaining the supernatant. Lysed fibroblast and muscle samples were incubated with dissociation buffer, containing $6.25 \mathrm{mM}$ Tris/HCl pH $6.8,2 \%$ SDS, $10 \%$ glycerol, $0.01 \%$ bromophenol blue and $100 \mathrm{mM}$ DTT, for $20 \mathrm{~min}$ at $37^{\circ} \mathrm{C}$.

All protein lysates were electrophoresed through $12 \%$ polyacrylamide resolving gels, cast, run and transferred on Bio-Rad Mini-Protean $®$ Tetra Cell systems, at 200 V. Target proteins were interrogated by incubation overnight with antibodies at $4{ }^{\circ} \mathrm{C}$ and the resulting signals were visualised with ECL-prime (GE Healthcare) using Image Lab software on a BioRad Chemidoc MP. Antibodies used were the following: SDHA (ab14715; Abcam), ATP5A (ab110273; Abcam), ATP5B (ab14730; Abcam), UQCRC2 (ab14745; Abcam), MT-CYB (55090-1-AP; Proteintech Europe), MT-COI (ab14705; Abcam), MT-COII (ab110258; Abcam), NDUFB8 (ab110242; Abcam) and GFM2 (ab74874; Abcam).

\section{$\left[{ }^{35} \mathrm{~S}\right]$ metabolic labelling}

Assessment of de novo mitochondrial protein synthesis was carried out in patient and control fibroblasts grown to a confluency of $80 \%$. Cells were washed three times for $10 \mathrm{~min}$ in methionine/cysteine-free media (Sigma, D0422) before adding $10 \%$ dialysed FCS (Sigma F0392) and $100 \mu \mathrm{g} / \mathrm{mL}$ emetine dihydrochloride to inhibit cytosolic translation. $20 \mu \mathrm{l}$ of $\left[{ }^{35} \mathrm{~S}\right]$-methionine/cysteine mix (Perkin-Elmer Easy Tag Express protein labelling mix, NEG-772, 73\% Lmet, $22 \% \mathrm{~L}$-cys) was added to each flask and cells were incubated at $37^{\circ} \mathrm{C}$ for $1 \mathrm{~h}$. Cells were then washed in standard growth media containing methionine and harvested in PBS + $1 \mathrm{mM}$ EDTA, pelleted at $200 \mathrm{~g}$ for $4 \mathrm{~min}$ and resuspended in PBS $+1 \mathrm{mM}$ PMSF and $1 \times$ EDTA-free protease inhibitor 
before being subjected to $15 \%$ SDS-PAGE as before. The gel was stained with Coomassie brilliant blue, fixed overnight (30\% methanol, 10\% acetic acid, 3\% glycerol) and vacuumdried prior to exposure using a PhosphorImage screen. Signal was detected using the Typhoon FLA9500 Phosphorimager and ImageQuant software (GE Healthcare) and mtDNAencoded proteins were assigned to corresponding bands with reference to Chomyn et al. [25].

\section{Results}

\section{Histopathological and biochemical studies}

The muscle morphology of Patient 1 revealed no marked structural abnormalities visible upon staining with $H \& E$ (Fig. 1g). Oxidative enzyme histochemistry showed intense succinate dehydrogenase (SDH) activity (Fig. 1h) and a generalised decrease in cytochrome $c$ oxidase (COX) activity across the biopsy (Fig. 1i). Sequential COX-SDH histochemistry confirmed widespread COX deficiency in the presence of normal SDH activity (Fig. 1j). The assessment of respiratory chain enzyme activities in muscle from Patient 1 revealed impairment of complex IV activity, while the activities of complexes I and II were both within normal limits (Fig. 1k). Interestingly, the fibroblast cells from this patient appeared to express a mild combined OXPHOS defect involving both complexes I and IV, albeit with residual enzyme activities of $\sim 60 \%$ of normal (Fig. 11).

\section{Variant identification, confirmation and segregation studies}

WES of Patient 1 identified compound heterozygous variants in the GFM2 gene, a c.569G>A single nucleotide substitution in exon 8 resulting in an arginine to glutamine missense change at residue 190 (p.(Arg190Gln)) and a c.636delA single nucleotide deletion in exon 9 producing a frameshift mutation and premature termination codon (p.(Glu213Argfs*3)) that can be classified as pathogenic without further work (Fig. 2a). WES of Patient 2 revealed a homozygous c. $275 \mathrm{~A}>\mathrm{C}$ variant in exon 5 of GFM2, predicted to cause a tyrosine to serine missense change at residue 92 (p.(Tyr92Ser)) (Fig. 2b). Sanger sequencing of the probands plus their respective parents confirmed the segregation of the bi-allelic GFM2 variants with disease in both families (Fig. 2a, b). All GFM2 variants have been submitted to ClinVar (https://www.ncbi.nlm.nih.gov/ clinvar/) with the following identifiers: c.569G $>\mathrm{A}$, SCV000605919; c.636delA, SCV000605920; c.275A>C, SCV000605921.

\section{Missense residue conservation and in silico predictions of pathogenicity}

Sequence conservation of each missense variant, along with the surrounding residues, showed that the p.Arg190 residue is conserved in mammals, but is less so in lower species. The region flanking the p.(Arg190Gln) change is moderately conserved (Fig. 2c). In contrast, the homozygous p.(Tyr92Ser) variant in Patient 2 involves a tyrosine that is conserved from humans through to yeast and the entire surrounding region is extremely well conserved (Fig. 2d).

All three GFM2 variants are extremely rare; the c.564A>G, p.(Arg190Gln) allele, harboured by Patient 1 , is present in 5/276,072 alleles on gnomAD $(\mathrm{MAF}=0.00002)$, while the c.275A $>$ C, p. $($ Tyr92Ser $)$ variant present in Patient 2 in a homozygous state is entirely novel. In silico tools produced mixed predictions - the p.(Tyr92Ser) missense variant was classified as likely to affect protein function according to all four in silico prediction tools. SIFT and aGVFD classified the p.(Arg190Gln) change in Patient 1 as unlikely to interfere with protein function while PolyPhen was strongly supportive of a deleterious effect. CADD scores were 35 for c. $564 \mathrm{~A}>\mathrm{G}$ and 28.7 for c. $275 \mathrm{~A}>\mathrm{C}$, predicting that these variants are in the top 0.01 and $0.1 \%$ of deleterious single nucleotide variants of the reference genome, respectively.

\section{Western blotting studies reveal varying OXPHOS defects}

Western blot analysis was performed on the fibroblast lysates from both patients to investigate the effect of the GFM2 variants on the steady-state levels of individual OXPHOS complex subunits. There was no apparent effect on the abundance of OXPHOS components in fibroblast cells from Patient 1 (Fig. 3a), consistent with the mild biochemical defects reported on direct enzyme assay. However, a clear decrease in steady-state levels of NDUFB8 (complex I), CYTB and CORE2 (complex III) and COXI and COXII (complex IV) was observed in the fibroblasts of Patient 2 compared to controls (Fig. 3a). In contrast to fibroblasts, skeletal muscle lysates from Patient 1 exhibited a marked decrease in steady-state levels of complex IV components, COXI and COXII (Fig. 3b). No skeletal muscle was available for Patient 2.

Investigation into the steady-state level of mtEFG2 was only possible following Western blot analysis of a skeletal muscle sample from Patient 1; fibroblast protein lysates repeatedly failed to produce a clear signal due to nonspecificity of the commercial antibody in this tissue. The level of mtEFG2 protein in skeletal muscle from Patient 1 was approximately $52 \%$ of controls when quantified using Image Lab ${ }^{\text {TM }}$ Software (Fig. 3b). 
Fig. 2 Segregation studies and missense residue conservation. a Familial pedigree and sequence data for Patient 1 and parents demonstrating recessive inheritance of compound heterozygous c. $569 \mathrm{G}>\mathrm{A}$, p.(Arg190Gln) and c.636delA, p.(Glu213Argfs*3) GFM2 variants. b Familial pedigree and sequence data for Patient 2 and parents demonstrating recessive inheritance of a homozygous c. $275 \mathrm{~A}>\mathrm{C}$, p.(Tyr92Ser) GFM2 variant. Multiple sequence alignment of GFM2 reveals moderate evolutionary conservation of the p.Arg 190 residue (c) and the p.Tyr92 residue is invariant (d) (both residues shown by an asterisk) a

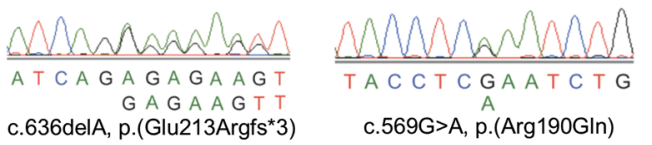

b

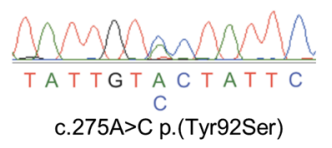

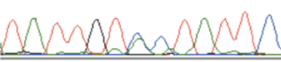

TA T TG T A C TA T T C c. $275 \mathrm{~A}>\mathrm{C}$ p. (Tyr92Ser)
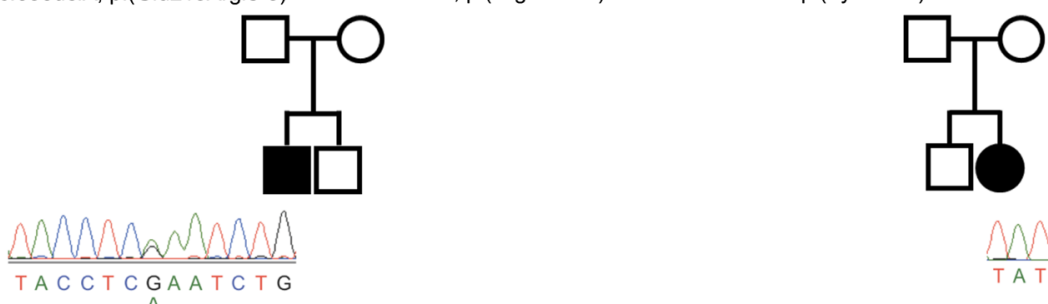

c.569G $>$ A, p. (Arg190GIn)

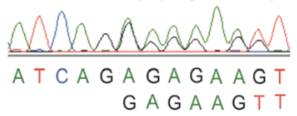

c.636delA, p.(Glu213Argfs*3)

c

$\begin{array}{lcc} & 557 & \\ \text { Variant } & \text { TVWRQADKHNIPQICFLNKMDKT } \\ \text { Human } & \text { TVWRQADKHNIPRI FLNKMDKT } \\ \text { Mouse } & \text { TVWRQADKHKI PRI FLNKMDKT } \\ \text { Platypus } & \text { TVWKQADKHHIPRI FLNKMDKT } \\ \text { Chicken } & \text { TVWRQADKHQI PRI FLNKMDKN } \\ \text { Frog } & \text { NSRRAASSFYRGRV QLHKIDHG } \\ \text { Zebrafish } & \text { TVWRQAEKHQI PCV FLNKMDKP } \\ \text { C. elegans } & \text { TVWRQSSKFKLAHFFINKMDKL }\end{array}$

d

\begin{tabular}{|c|c|}
\hline 26 & 63 \\
\hline ant & DAGKTTTTERILSYSGYTRSLGD \\
\hline Human & DAGKTTTTERILYYS $G Y T R S L G D$ \\
\hline buse & DAGKTTTTERILYYS $G Y T R S L \in D$ \\
\hline Latypus & DAGKTTTTERILYYS $G Y T R S L G D$ \\
\hline Chicken & DAGKTTTTERMLYYS $G Y I R T L G D$ \\
\hline Fre & $D A G K T T T T E R M L Y Y S G Y I R T L G D$ \\
\hline zebraf & $D A G K T T T T E R M L Y Y A G K T R A L G E$ \\
\hline 7 & $D A G K T T V T E R L L Y L A \in A I H V A \in H$ \\
\hline
\end{tabular}

a

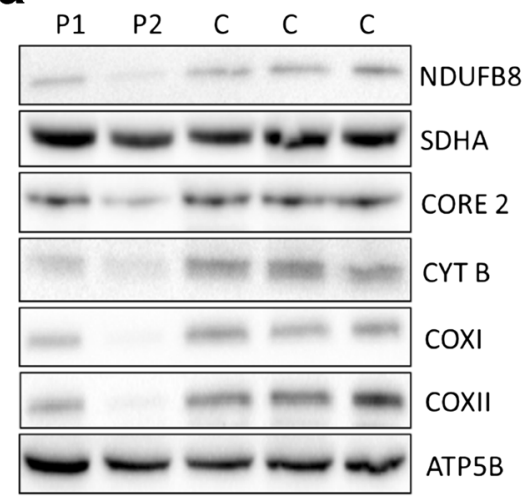

b

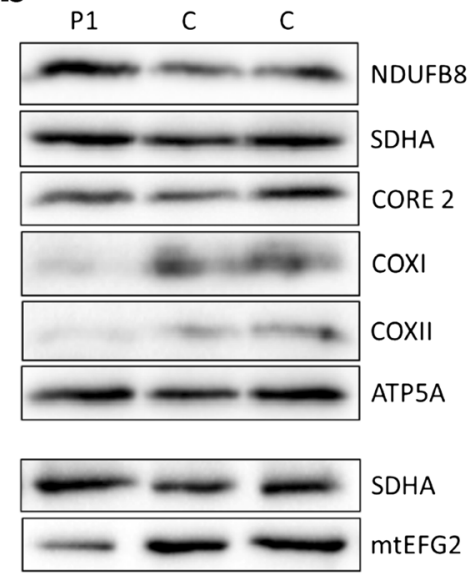

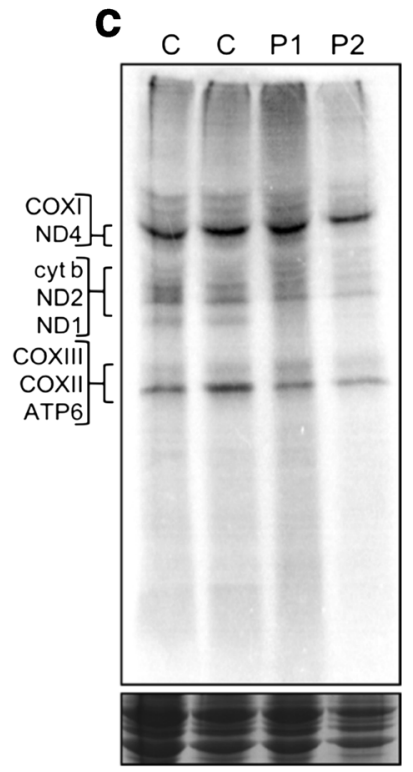

Fig. 3 Western blot studies and a $\left[{ }^{35} \mathrm{~S}\right]$ translational assay give insight into molecular effect of GFM2 variants. a Fibroblast Western blot panel, with SDHA as a loading control. The panel demonstrates decreased steady-state levels of NDUFB8 (complex I), CORE 2 and CYT B (complex III) and COX I and COX II (complex IV) in the fibroblasts of Patient 2, but unchanged levels in the fibroblasts of Patient 1. b Skeletal muscle Western blot panel for Patient 1, with SDHA as a loading control.
A complex IV deficiency is apparent, with decreased steady-state levels of COX I and COX II. Steady-state levels of other OXPHOS subunits remain unchanged. Levels of $\mathrm{mtEFG} 2$ protein are $\sim 50 \%$ of controls. $\mathrm{c}$ $\left[{ }^{35} \mathrm{~S}\right]$ methionine/cysteine incorporation in growing fibroblasts as a measure of de novo mitochondrial protein synthesis showed no difference between either Patient 1 or Patient 2 and the controls using Coomassie stain as loading control (bottom panel) 


\section{$\left[{ }^{35} \mathrm{~S}\right]$ translation assay}

To assess whether the GFM2 variants present in Patient 1 and Patient 2 cause any impairment of de novo mitochondrial protein synthesis in growing fibroblast cells, a $\left[{ }^{35} \mathrm{~S}\right]$ metabolic labelling assay was performed. In the case of both Patient 1 and Patient 2, there does not appear to be any effect on the incorporation of $\left[{ }^{35} \mathrm{~S}\right]$ methionine/cysteine into newly synthesised mitochondrial DNA-encoded proteins (Fig. 3c).

\section{Discussion}

Through the application of whole exome sequencing, we have identified previously unpublished recessive GFM2 variants in two unrelated patients with clinical features of mitochondrial disease and biochemical evidence of respiratory chain dysfunction. Western blot analysis of patient fibroblasts showed differential effects at the protein level for each of these patients. While Patient 2 shows decreased steady-state levels of mitochondrial-encoded OXPHOS subunits NDUFB8 (of complex I), COXI/COXII (of complex IV) and CYTB/CORE2 (of complex III), indicating a combined OXPHOS deficiency, steady-state OXPHOS protein levels were relatively unchanged in Patient 1 when compared to controls (Fig. 3a).

The gene GFM2, located at 5q13.3, encodes the protein mtEFG2 which has an essential role following the termination of mitochondrial translation as GTP hydrolysis is necessary for the recycling of the mitoribosome [17]. To date, GFM2 variants have been identified in four patients (two sets of siblings), with phenotypes described as microcephaly, simplified gyral pattern and insulin-dependent diabetes in the first report and Leigh syndrome complicated by arthrogryposis multiplex congenita in the most recent family [20, 21]. While Leigh syndrome is a familiar mitochondrial phenotype, there are clinical aspects to these four cases that are rather atypical for mitochondrial disease, simplified gyral pattern and arthrogryposis multiplex. In contrast, the two cases reported here have a consistent clinical phenotype that is entirely compatible with mitochondrial disease and includes 'red flag' clinical features such as neurodevelopmental regression.

The steady-state levels of COXI and COXII in the skeletal muscle of Patient 1 were decreased (Fig. 3b), demonstrating a complex IV deficiency. These results are in accordance with the original diagnostic assessment of individual respiratory chain enzyme activities in Patient 1, which detected a complex IV activity of around $40 \%$ of controls. The residual levels of mtEFG2 in skeletal muscle from Patient 1 were approximately $50 \%$ of the two controls (Fig. 3b). The c.636delA p.(Glu213Argfs*3) GFM2 mutation in Patient 1 causes a frame shift and a premature stop codon and is, therefore, likely to encode a non-functional truncated version of mtEFG2 that is likely to undergo nonsensemediated mRNA decay. The 50\% residual level of steady-state
mtEFG2 seen in the skeletal muscle of Patient 1 can be explained by the $p .(\operatorname{Arg} 190 \mathrm{Gln})$ variant occurring in a region of the gene with only moderate sequence conservation. This is supported by the in silico pathogenicity predictions suggesting the missense change would not have major impacts on protein stability, consistent with the loss of only $48 \%$ normal mtEFG2 levels.

Steady-state OXPHOS subunit deficiency in Patient 1 is observable in skeletal muscle, but not fibroblasts, indicating tissue-specific differences. The deficiency of complex IV activity was most severe in the muscle (Fig. 1k) and less so in fibroblasts (Fig. 11). This tissue-specific discrepancy in severity of OXPHOS defect is not uncommon in defects of mitochondrial translation and can arise due to the differences in ATP reliance in either tissue or differential levels of gene expression in the two tissue types. A number of deficiencies due to mt-aaRS mutations, such as YARS2 encoding the mitochondrial tyrosyl-tRNA synthetase, show a much clearer OXPHOS defect in myoblasts than fibroblasts. In the case of pathogenic YARS2 variants, a marked decrease in steady-state levels of NDUFB8 was observed only in myoblasts [26]. Muscle relies much more heavily on the generation of ATP through oxidative phosphorylation, and so variants in mitochondrial disease genes can on occasion present a much clearer effect at the steady-state protein level [27].

The clear difference in severity of OXPHOS defect between Patient 1 and Patient 2, visible upon Western blotting, can be explained by considering both the level of sequence conservation at the missense variant sites and in silico predictions of pathogenicity. There is consensus across two out of four prediction programmes (SIFT and aGVGD) that the p.(Arg190Gln) missense variant present in Patient 1 has a milder consequence compared to the p.(Tyr92Ser) GFM2 variant of Patient 2. Despite Patient 1 having degradation of mtEFG2 from the allele containing the p.(Glu213Argfs*3) frameshift mutation, if the p.(Arg190Gln) amino acid change is less damaging and the remaining $\mathrm{mtEFG} 2$ protein retains some function, then it might be expected that the OXPHOS phenotype is less severe.

Despite $\mathrm{mtEFG} 2$ playing an important role in the termination step of mitochondrial translation, our $\left[{ }^{35} \mathrm{~S}\right]$ translational assay showed no marked decrease in $\left[{ }^{35} \mathrm{~S}\right]$ methionine/ cysteine incorporation in growing fibroblasts as a measure of de novo mitochondrial protein synthesis, which may have been expected for Patient 1, due to a lack of clear phenotype in fibroblast cells. A possible explanation for the signal for Patient 2 being similar to controls can be found when the stage of translation at which $\mathrm{mtEFG} 2$ exerts its function is considered. In the majority of cases, mitoribosome recycling occurs upon completion of synthesis of a nascent peptide [28]. If mtEFG2 is dysfunctional, it will not prevent the incorporation of amino acids throughout the synthesis of a peptide, but will prevent the proper release of this product. Therefore, the signal coming from incorporated methionine/cysteine will not differ between control and patient cells. 
In summary, we present two patients with developmental delay, dystonia, dysarthria and neuroimaging abnormalities in the putamen and caudate nuclei, along with subcortical white matter involvement who harbour previously unreported GFM2 variants, identified through the application of WES. The histochemical, biochemical and genetic investigations, together with the functional data presented in this report, form compelling evidence that the GFM2 variants identified through WES are pathogenic and causative of mitochondrial disease in both patients.

Funding information This work is funded by the Wellcome Centre for Mitochondrial Research (203105/Z/16/Z), the Medical Research Council (MRC) Centre for Translational Research in Neuromuscular Disease, Mitochondrial Disease Patient Cohort (UK) (G0800674), the Lily Foundation, the UK NHS Highly Specialised Service for Rare Mitochondrial Disorders of Adults and Children and the Department of Health via the NIHR comprehensive Biomedical Research Centre award to Guy's and St. Thomas' NHS Foundation Trust in partnership with the King's College London. CLA was supported by an NIHR/CSO Healthcare Science Research Fellowship from the National Institute for Health Research (NIHR-HCS-D12-03-04). TBH was supported by the German Federal Ministry of Education and Research (BMBF) within the framework of the e:Med research and funding concept (grant no. FKZ 01ZX1405C). This study was also supported by the German Bundesministerium für Bildung und Forschung (BMBF) and Horizon 2020 through E-Rare project GENOMIT (01GM1603, 01GM1207 to H.P.) and the EU Horizon 2020 Collaborative Research Project SOUND (633974 to H.P.).

Compliance with ethical standards All studies were completed according to local Ethical Approval of the Institutional Review Boards of Newcastle University (the National Research Ethics Service Committee North East-Newcastle \& North Tyneside 1) and of the Technische Universität München. In agreement with the Declaration of Helsinki, all individuals or their guardians provided written informed consent before undergoing evaluation and genetic testing.

Conflict of interest The authors declare that there is no conflict of interest.

Open Access This article is distributed under the terms of the Creative Commons Attribution 4.0 International License (http:// creativecommons.org/licenses/by/4.0/), which permits unrestricted use, distribution, and reproduction in any medium, provided you give appropriate credit to the original author(s) and the source, provide a link to the Creative Commons license, and indicate if changes were made.

\section{References}

1. Anderson S, Bankier AT, Barrell BG et al (1981) Sequence and organization of the human mitochondrial genome. Nature 290:457-465

2. Wallace DC (1989) Mitochondrial DNA mutations and neuromuscular disease. Trends Genet 5:9-13

3. Taylor RW, Pyle A, Griffin H et al (2014) Use of whole-exome sequencing to determine the genetic basis of multiple mitochondrial respiratory chain complex deficiencies. JAMA 312:68-77. https:// doi.org/10.1001/jama.2014.7184

4. Sissler M, González-Serrano LE, Westhof E (2017) Recent advances in mitochondrial aminoacyl-tRNA synthetases and disease. Trends Mol Med 23:693-708. https://doi.org/10.1016/j.molmed.2017.06.002
5. Pearce S, Nezich CL, Spinazzola A (2013) Mitochondrial diseases: translation matters. Mol Cell Neurosci 55:1-12. https://doi.org/10. 1016/j.men.2012.08.013

6. Alston CL, Rocha MC, Lax NZ et al (2017) The genetics and pathology of mitochondrial disease. J Pathol 241:236-250. https://doi.org/10.1002/path.4809

7. Andersson SG, Zomorodipour A, Andersson JO et al (1998) The genome sequence of Rickettsia prowazekii and the origin of mitochondria. Nature 396:133-140. https://doi.org/10.1038/24094

8. O'Brien TW (1971) The general occurrence of $55 \mathrm{~S}$ ribosomes in mammalian liver mitochondria. J Biol Chem 246:3409-3417

9. Kılıç M, Oğuz K-K, Kılıç E et al (2017) A patient with mitochondrial disorder due to a novel mutation in MRPS22. Metab Brain Dis 32:1389-1393. https://doi.org/10.1007/s11011-017-0074-5

10. Miller C, Saada A, Shaul N et al (2004) Defective mitochondrial translation caused by a ribosomal protein (MRPS16) mutation. Ann Neurol 56:734-738. https://doi.org/10.1002/ana.20282

11. Galmiche L, Serre V, Beinat $M$ et al (2011) Exome sequencing identifies MRPL3 mutation in mitochondrial cardiomyopathy. Hum Mutat 32:1225-1231. https://doi.org/10.1002/humu.21562

12. Soiferman D, Ayalon O, Weissman S, Saada A (2014) The effect of small molecules on nuclear-encoded translation diseases. Biochimie 100:184-191. https://doi.org/10.1016/j.biochi.2013.08.024

13. Diodato D, Ghezzi D, Tiranti V (2014) The mitochondrial aminoacyl tRNA synthetases: genes and syndromes. Int J Cell Biol 2014:787956-787911. https://doi.org/10.1155/2014/787956

14. Musante L, Püttmann L, Kahrizi K et al (2017) Mutations of the aminoacyl-tRNA-synthetases SARS and WARS2 are implicated in the etiology of autosomal recessive intellectual disability. Hum Mutat 38:621-636. https://doi.org/10.1002/humu.23205

15. Hammarsund $M$, Wilson $W$, Corcoran $M$ et al (2001) Identification and characterization of two novel human mitochondrial elongation factor genes, hEFG2 and hEFG1, phylogenetically conserved through evolution. Hum Genet 109:542-550. https://doi.org/10. 1007/s00439-001-0610-5

16. Zhang D, Yan K, Zhang Y et al (2015) New insights into the enzymatic role of EF-G in ribosome recycling. Nucleic Acids Res 43: 10525-10533. https://doi.org/10.1093/nar/gkv995

17. Tsuboi M, Morita H, Nozaki Y et al (2009) EF-G2mt is an exclusive recycling factor in mammalian mitochondrial protein synthesis. Mol Cell 35:502-510. https://doi.org/10.1016/j.molcel.2009.06.028

18. Brito S, Thompson K, Campistol J et al (2015) Corrigendum: longterm survival in a child with severe encephalopathy, multiple respiratory chain deficiency and GFM1 mutations. Front Genet 6:254. https://doi.org/10.3389/fgene.2015.00254

19. Simon MT, Ng BG, Friederich MW et al (2017) Activation of a cryptic splice site in the mitochondrial elongation factor GFM1 causes combined OXPHOS deficiency. Mitochondrion 34:84-90. https://doi.org/10.1016/j.mito.2017.02.004

20. Fukumura S, Ohba C, Watanabe T et al (2015) Compound heterozygous GFM2 mutations with Leigh syndrome complicated by arthrogryposis multiplex congenita. J Hum Genet 60:509-513. https://doi.org/10.1038/jhg.2015.57

21. Dixon-Salazar TJ, Silhavy JL, Udpa N et al (2012) Exome sequencing can improve diagnosis and alter patient management. Sci Transl Med 4:138ra78-138ra78. https://doi.org/10.1126/scitranslmed.3003544

22. Kirby DM, Thorburn DR, Turnbull DM, Taylor RW (2007) Biochemical assays of respiratory chain complex activity. Methods Cell Biol 80:93-119. https://doi.org/10.1016/S0091679X(06)80004-X

23. Thompson K, Majd H, Dallabona $\mathrm{C}$ et al (2016) Recurrent de novo dominant mutations in SLC25A4 cause severe early-onset mitochondrial disease and loss of mitochondrial DNA copy number. Am J Hum Genet 99:1405. https://doi.org/10.1016/j.ajhg.2016.11.001 
24. Kremer LS, Bader DM, Mertes C et al (2017) Genetic diagnosis of Mendelian disorders via RNA sequencing. Nat Commun 8:15824. https://doi.org/10.1038/ncomms15824

25. Chomyn A (1996) In vivo labeling and analysis of human mitochondrial translation products. Meth Enzymol 264:197-211

26. Sommerville EW, Ng YS, Alston CL et al (2017) Clinical features, molecular heterogeneity, and prognostic implications in YARS2related mitochondrial myopathy. JAMA Neurol. https://doi.org/ 10.1001/jamaneurol.2016.4357
27. Scarpelli M, Todeschini A, Volonghi I et al (2017) Mitochondrial diseases: advances and issues. Appl Clin Genet 10:21-26. https:// doi.org/10.2147/TACG.S94267

28. Chrzanowska-Lightowlers ZMA, Pajak A, Lightowlers RN (2011) Termination of protein synthesis in mammalian mitochondria. J Biol Chem 286:34479-34485. https://doi.org/10.1074/jbc.R111. 290585 